\title{
EL PAPEL DE LAS CIUDADES MEDIAS DE INTERIOR EN EL DESARROLLO REGIONAL. EL CASO DE ANDALUCÍA
}

\author{
Marco Garrido Cumbrera \\ Departamento de Geografía Física y Análisis Geográfico Regional. Universidad de Sevilla \\ mcumbrera@us.es \\ Juan Carlos Rodríguez Mateos \\ Departamento de Geografía Humana. Universidad de Sevilla \\ juancarlos@us.es \\ Enrique López Lara \\ Departamento de Geografía Física y Análisis Geográfico Regional. Universidad de Sevilla \\ elopezl@us.es
}

\section{RESUMEN}

Las ciudades medias presentan ventajas frente a las grandes urbes y, por ello, deberían desempeñar funciones esenciales para el equilibrio territorial. Sin embargo, en los últimos tiempos, las Administraciones Públicas han destinado más inversiones a las grandes metrópolis, provocando una concentración demográfica, económica, de infraestructuras y equipamientos en éstas, con la consiguiente pérdida del protagonismo que las ciudades medias tenían décadas atrás. En el presente artículo se establecen tipologías de ciudades medias andaluzas en función de su localización para evaluar su evolución en los últimos tiempos.

Palabras clave: sistema de ciudades, ciudades medias de interior, áreas metropolitanas, equilibrio territorial, Andalucía.

\section{ABSTRACT}

Mid-size towns and cities have certain advantages over large cities and ought to play essential roles in spatial balance. However, in recent times the Public Administration has

Fecha de recepción: octubre 2014.

Fecha de aceptación: septiembre 2015. 
allocated more investments to large metropolises, leading to demographic, economic and infrastructural and equipment concentration in these, and mid-size towns and cities consequently relinquishing the leadership that they used to have several decades ago. This article discusses some of the main types of Andalusian mid-size towns and cities on the basis of their location and the different ways that they have developed in recent times.

Keywords: city system, inland mid-size towns and cities, metropolitan areas, spatial balance, Andalusia.

\section{PAPEL DE LAS CIUDADES MEDIAS EN EL EQUILIBRIO TERRITORIAL}

Frente al auge de las grandes ciudades, existe una gran tradición en la promoción de las ciudades medias por su capacidad para favorecer el desarrollo regional equilibrado. En este sentido, son destacables los planteamientos del Banco Mundial y, sobre todo, del Observatorio Europeo en Red de la Ordenación del Territorio de la UE (ESPON), que publicó en 2006 un estudio titulado El Papel de las Ciudades de Tamaño Pequeño y Mediano en Europa (ESPON, 2006), además de financiar el proyecto TOWN - Small and Medium-Sized Towns (2011-2014), cuyo objetivo ha sido estudiar el papel de las ciudades pequeñas y medias, identificadas como aquellas ciudades con población entre los 5.00050.000 habitantes, en el desarrollo territorial equilibrado (ESPON, 2012). En los últimos años ha existido un prolífico interés científico por este tipo de ciudades en cuestiones de ordenación territorial, ya que poseen una gran capacidad para promover el desarrollo rural y regional, reducir la pobreza y atenuar la migración hacia las grandes urbes (Pasciaroni, 2012).

En muchos países (entre ellos España), la urbanización, la terciarización económica y la globalización, además de ciertas decisiones políticas, han dado lugar a que las grandes ciudades consigan mejores perspectivas de desarrollo, absorbiendo población y actividades económicas y, por tanto, recursos (López Lara, 2015). El desarrollo de macrocefalias metropolitanas, basadas en la concentración de población, actividades económicas, infraestructuras, equipamientos..., deriva en la pérdida del protagonismo que las ciudades medias tenían décadas atrás, transformándose las jerarquías de los sistemas urbanos tradicionales. Dichos procesos han comportado una creciente concentración de la toma de decisiones en unos pocos centros y, por consiguiente, la marginalización de centros secundarios o insuficientemente conectados a las redes mundiales (Vilagrasa Ibarz y Ganau Casas, 2003).

Una de las primeras consecuencias de la globalización es la diversificación de la producción de las grandes ciudades a través de las actividades de I+D, convirtiéndolas en incubadoras de nuevas firmas y nuevos productos e intensificando las diferencias entre las grandes urbes, las ciudades medias y el mundo rural. No obstante, a partir del surgimiento del paradigma de la globalización y de las nuevas tecnologías, la posición que ocupa cada ciudad en las nuevas redes no depende exclusivamente de su tamaño, sino de la capacidad para integrarse en el espacio de flujos de bienes, servicios, personas e información (Pasciaroni, 2012). Es por ello que factores tradicionales de localización (acceso a los recursos naturales, cercanía a los grandes centros de consumo) prácticamente han perdido importancia; el avance en transportes y comunicaciones permite a las empresas 
ubicarse en lugares alejados de donde se concentran los recursos físicos o el mercado y seguir manteniendo ventajas comparativas y competitivas (Macías Macías, 2004). Con estas transformaciones, las ciudades medias también experimentan modificaciones en sus funciones, dado que, no solo actúan como nexo entre niveles urbanos superiores e inferiores, sino que establecen relaciones con otras ciudades y territorios del mismo nivel jerárquico, articulándose en redes dentro de un espacio integrado a escala nacional e internacional (Marques Da Costa, 2002). Además, las ciudades medias se convierten en centros urbanos con suficiente masa crítica y con voluntad de actuar como intermediarios entre la gran ciudad y los espacios rurales, al ser capaces de generar conocimiento y desarrollo en su entorno próximo y de equilibrar el territorio frente a las macrocefalias metropolitanas (Vilagrasa Ibarz, 2000).

La constatación de estos fenómenos ha impulsado una preocupación por el abandono del mundo rural y las ciudades medias en el marco de la globalización (Macías Macías, 2004). El mercado laboral pierde su atractivo, aumentando el fracaso escolar y el desempleo, especialmente en los grupos de edad más jóvenes, que emigran a las áreas metropolitanas (con mercados laborales más diversificados), quedando una población envejecida y poco cualificada (García Sanz y Martínez Paricio, 1998). Los servicios públicos se van reduciendo y se deterioran las infraestructuras, debido principalmente a las limitaciones financieras (se cierran servicios educativos, sanitarios, empresas privadas...) (Sáez Pérez et al., 2001), erosionándose la base local, aumentando las desigualdades socio-económicas y produciéndose la marginación de las zonas rurales. Este proceso, además, genera que se congestionen las áreas metropolitanas sin la deslocalización necesaria que pueden proporcionar las ciudades medias. Funciones como la residencial o el desarrollo de actividades económicas necesitan de un sistema de ciudades equilibrado, apoyado además en el menor coste del suelo periférico frente al propiamente urbano y central, aspectos que deben ser potenciados con una red de transportes nacional y europea -actualmente deficitaria- a través de una fuerte inversión.

En suma, las ciudades medias articulan el territorio y funcionan como centros de referencia para un territorio más o menos inmediato, ofreciendo ventajas respecto a las grandes metrópolis urbanas al configurarse como (Llop Torné, 1999): i) sistemas más equilibrados y sostenibles por razones de escala, que ejercen relaciones más armónicas, abiertas y equilibradas con su territorio; ii) centros más fácilmente gobernables, gestionables y controlables, que suelen permitir una mayor participación ciudadana en el gobierno y gestión de la ciudad; iii) asentamientos con escalas y dimensiones más humanas que ayudan al ciudadano a identificarse más con su ciudad al recibir mayor apoyo en las relaciones interpersonales directas; iv) ciudades que no poseen los problemas ambientales que presentan las grandes metrópolis urbanas.

Sin embargo, las ciudades medias también poseen algunas desventajas frente a las grandes metrópolis urbanas, ya que, por un lado, y debido a su tamaño, tienen una menor diversidad social y cultural y se produce en ellas una cierta endogamia social, con menor cualificación de sus recursos humanos que, en muchos casos, se han desplazado hacia las grandes urbes; por otro, presentan menor competitividad económica, con menor acceso a los principales flujos de información y capital, frente a las metrópolis urbanas, que tienden a concentrar las principales funciones del sistema. 


\section{CLASIFICACIÓN DE LAS CIUDADES MEDIAS A PARTIR DE DISTINTOS CRITERIOS}

\section{II.1. En función del tamaño poblacional}

Una de las razones para explicar la complejidad de estudiar el papel e importancia de las ciudades medias radica en la dificultad de su definición (Andrés López, 2008). La delimitación de las posiciones intermedias debe partir de los extremos superior e inferior de una jerarquía, es decir, necesita una contextualización. A nivel mundial encontramos importantes diferencias, por lo que no resulta posible utilizar los mismos rangos cuantitativos para definir las posiciones intermedias en diferentes contextos. El término ciudad media es, por tanto, un término complejo en cuanto a sus definiciones, aceptándose en el caso español cierto grado de arbitrariedad (Esteban y López, 1989; Vilagrasa Ibarz, 1999).

Desde la perspectiva puramente cuantitativa, la principal variable para definir ciudades medias suele ser el tamaño poblacional o número de habitantes. A pesar de su aparente objetividad, los rangos cambian según el contexto (20.000-500.000 en Europa, 25.000-100.000 en Pakistán, hasta 1 millón en Argentina, etc.) (Llop Torné, 1999). La Unión Europea plantea los límites entre los 50.000 y los 500.000 habitantes (Bellet Sanfeliu, Llop Torné, 1999). La definición más común de ciudad media es la de una ciudad de entre 20.000 y 200.000 habitantes, dependiendo de la densidad de población y del sistema urbano del país (European Foundation for the Improvement of Living and Working Conditions, 1994; Rivkin y Rivkin, 1982; Rondinelli, 1983).

\section{II.2. Según las funciones que desempeñan}

El criterio demográfico no constituye el único factor, ni siquiera el más decisivo, utilizado para definir a las ciudades medias si se tienen en cuenta los múltiples roles y las funciones asociadas a las mismas (Satterthwaite y Tacoli, 2003). Las ciudades medias se definen como asentamientos urbanos de tamaño medio que desempeñan funciones urbanas, tales como la intermediación entre centros de distintos niveles jerárquicos (Sposito, 2004). Si bien en un primer momento las ciudades medias fueron definidas de una manera demasiado cuantitativa, actualmente localización, identidad urbana, usos del suelo, funciones urbanas e incluso actividades económicas son los elementos que determinan si un núcleo es una ciudad media o no (Rodríguez Martínez y Sánchez Escolano, 2010a). Por otra parte, es fundamental la función que la ciudad desempeña en su territorio circundante, la influencia que ejerce y mantiene en éste, y los flujos y relaciones que genera hacia el exterior (Pasciaroni, 2012). Desde la óptica clásica de los estudios geográficos, se ha considerado la ciudad media en relación con un territorio o región que lidera (Vilagrasa Ibarz y Ganau Casas, 2003), no coincidiendo siempre la noción de ciudad media en cuanto a tamaño con la de ciudad media en sentido funcional.

Algunos autores señalan que las funciones de las ciudades medias están relacionadas con la articulación territorial y la provisión de bienes y servicios a los territorios en los que ejercen influencia, jugando un papel distribuidor de productos derivados de los asentamientos rurales. Debemos añadir a ello: la función administrativa (suelen ser el emplazamiento de órganos administrativos locales) (Pasciaroni, 2012); su papel como motor de desarrollo 
territorial regional (oferta de servicios a la ciudadanía, empresas locales y grandes empresas) (Graña, 2002; Kunzmann, 2010); deslocalización empresarial, debido al menor precio de sus suelos (García Montalvo, 2006); la función residencial en el seno de áreas metropolitanas (verdaderas «islas paraíso» que aúnan lo urbano y lo rural) (Entrena Durán, 2004) y la función de descongestión (sede de funciones descentralizadas desde el centro metropolitano) (Kuzmann, 2010).

Sobre la base de la experiencia francesa, algunos autores señalan que los roles que deben cumplir las ciudades medias en el territorio son: constituirse como centros de empleo industrial y terciario alternativos a las grandes ciudades para la población que emigra desde territorios rurales circundantes; ofrecer alojamiento y equipamiento colectivo a la población que llega y establece su residencia en la ciudad; disponer de servicios públicos capaces de ofrecer calidad de vida urbana a sus habitantes; poseer medios de comunicación que aseguren su conectividad con los niveles más elevados de la jerarquía urbana y con el espacio rural próximo, evitando la exclusión de este espacio (Lajugie, 1974; Amorim Filho y Serra, 2001). En general, las ciudades medias poseen una combinación de funciones de abastecimiento, desarrollo y descongestión de la región en la que se encuentran situadas.

\section{II.3. En función de su localización geográfica}

La localización geográfica, así como la posición intermedia de las ciudades dentro de la red urbana, constituyen atributos de igual o mayor importancia que el tamaño poblacional en la caracterización de las mismas (Amorim Filho y Rigotti, 2002). En opinión de Kunzmann (2010), en Europa las ciudades medias pueden localizarse: a) dentro de las áreas metropolitanas; b) cercanas a las áreas metropolitanas; c) alejadas de las áreas metropolitanas; d) en una frontera. Dentro de estas tipologías de ciudades medias existen diferencias derivadas de las ventajas de su locación concreta, activos locales, tradiciones culturales, límites adyacentes, o incluso factores político-administrativos relacionados con acontecimientos históricos ocurridos durante siglos. En cualquier caso, la creciente concentración del desarrollo económico en las regiones metropolitanas afecta a cada una de las tipologías de ciudades medias de manera distinta.

\section{II.3.1. Situadas dentro de las áreas metropolitanas}

Las ciudades medias que se localizan dentro de las regiones metropolitanas, y que fueron antiguos pueblos rurales, han crecido rápidamente en las últimas décadas debido a la expansión urbana y a la presión del desarrollo. Este tipo de ciudades medias son las que más se han beneficiado de las actuales tendencias del desarrollo territorial, ofreciendo una combinación de las ventajas de vivir en el centro metropolitano y en el campo. Por lo general, estas ciudades medias tienen una larga historia, fuerte identidad y un alto nivel de vida, lo que se refleja en tradiciones locales profundamente arraigadas, buenas escuelas y servicios públicos, alto grado de seguridad, accesibilidad a la naturaleza, posibilidades de ocio y viviendas relativamente asequibles. Su excelente conectividad por carretera y ferrocarril las convierte en ciudades dormitorio para la población metropolitana que busca un estilo de vida tradicional o vivienda barata; su acceso al aeropuerto metropolitano posibilita los viajes de negocios. 
Son consideradas localizaciones atractivas para los servicios producidos fuera de la ciudad central y para las empresas que buscan localizaciones asequibles para sus oficinas secundarias o nuevas áreas de inversión. Por regla general, estas ciudades de tamaño medio crecen demográficamente y alcanzan cierto desarrollo económico. Con presupuestos saneados, pueden darse el lujo de mantener un alto nivel de infraestructura pública. La gestión pública es eficiente y las asociaciones público-privadas pueden organizarse adecuadamente.

\section{II.3.2. Situadas cercanas a las áreas metropolitanas}

Estas ciudades se encuentran en una posición diferente. Pueden estar vinculadas al núcleo por eficientes servicios de transporte o buenas conexiones a la red de autopistas metropolitanas. En estas condiciones, funcionan como una isla extraterritorial fuera de la región metropolitana en un entorno rural, con todas sus comodidades naturales, atracciones y potencialidades ambientales, al tiempo que las atracciones urbanas del núcleo metropolitano están accesibles en menos de una hora de trayecto. Cuando las conexiones con el centro metropolitano son adecuadas, se convierten en núcleos atractivos por los menores precios inmobiliarios y la diversidad de oportunidades de empleo.

\section{II.3.3. Ciudades medias alejadas de las áreas metropolitanas}

Localizadas lejos de las áreas metropolitanas, resultan menos atractivas para los hogares y las empresas, por lo que la población económicamente más activa tiende a abandonarlas (quedando allí la población envejecida y menos cualificada) y las infraestructuras públicas se van deteriorando. El lobbying por una mejor conectividad física al núcleo metropolitano se plantea como oportunidad para revertir la tendencia negativa a largo plazo, movilizando el capital territorial endógeno.

Las ciudades medias de la periferia europea son las perdedoras en la globalización. Su conectividad a la red de transporte nacional y europea es deficiente; la economía local sufre la desventaja de su localización y se realiza muy poca inversión interna y, cuando ésta se produce, se debe a enormes subvenciones públicas y ayudas para atraer inversores. En consecuencia, el mercado laboral pierde su atractivo y disminuyen las oportunidades de formación de jóvenes, que abandonan la escuela. El desempleo de larga duración aumenta a medida que los hogares jóvenes, económicamente activos, emigran a regiones metropolitanas que poseen mercados de trabajo más diversificados. Por otro lado, la población va envejeciendo y los centros educativos se cierran, reduciéndose la variedad y las posibilidades de elección. La base local se va erosionando. Debido a las contracciones económicas los servicios públicos se van reduciendo. Poco a poco, las desigualdades locales sociales y económicas aumentan, unidas a tensiones sociales y problemas de seguridad.

En Europa se observa cómo las ciudades medias alejadas de las regiones metropolitanas sufren los efectos de la actual fiebre metropolitana. A pesar del discurso político y los esfuerzos europeos para promover la cohesión territorial, las áreas que se encuentran situadas fuera de los límites de las regiones metropolitanas continúan sufriendo los efectos de las fuerzas globalizadoras y la competencia regional. Las regiones y pueblos situados en la periferia europea, fuera de las aglomeraciones urbanas, tienen cada vez más dificultades para mante- 
ner su desarrollo económico y sus funciones sociales y culturales. Dichas ciudades medias se ven afectadas por una economía global cada vez más competitiva. Con el fin de garantizar el empleo y mantener las funciones de servicio a una población estancada, estas ciudades medias se ven obligadas a encontrar su propio perfil entre la orientación internacional y el arraigo local.

\section{ORIGEN Y EVOLUCIÓN DE LA CIUDAD MEDIA ANDALUZA. SU PAPEL EN LA ORDENACIÓN TERRITORIAL}

\section{III.1. Introducción al papel de las ciudades medias andaluzas}

Las ciudades medias en Andalucía son piezas fundamentales en el modelo de asentamientos tradicional polinuclear, donde la ausencia histórica de una metrópolis regional impulsó el desarrollo de asentamientos medios. A ello hay que añadir la forma de ocupación del territorio. Desde la Edad Media, ha primado el poblamiento concentrado frente a la dispersión por motivos de seguridad y defensa; la continua inestabilidad (tierra de frontera) favoreció las grandes concentraciones de población en un número reducido de ciudades. Por otra parte, el sistema de gran propiedad agraria predominante en nuestra región (el latifundio), proporcionó un factor de desarrollo urbano importante, al estar basado en la utilización masiva de jornaleros que no poseían tierras y se veían obligados a concentrarse en ciudades (Díaz Quidiello, 2007). La relevancia de las ciudades medias andaluzas, no obstante, ha ido variando según las épocas históricas. Previamente a la provincialización de 1833, las ciudades medias tenían una gran importancia, al ser verdaderas capitales comarcales que controlaban el territorio y que alcanzaban cifras de población cercanas a las de algunas capitales de provincia (Serrano Martínez, 2005). Sin embargo, el proceso de provincialización impulsó las capitales provinciales, que se convirtieron en centros gravitatorios de las regiones y de conexión con el gobierno central, produciéndose cierto abandono y pérdida de influencia de las ciudades medias (Cano García, 2008). A pesar de ello, poco a poco fueron recuperándose, debido a su peso demográfico y a la tradicional importancia que poseían, siendo escogidas frecuentemente como sedes administrativas, o convirtiéndose en centros de desarrollo de nuevas actividades (industriales y de servicios), dejando patente la heterogeneidad del sistema urbano andaluz (Serrano Martínez, 2005).

Esta relativa recuperación quedó frenada cuando determinadas circunstancias hicieron entrar en crisis a estas ciudades. La emigración y la transformación del mundo rural tras el despegue industrial crearon una nueva potenciación de los grandes núcleos metropolitanos, que fueron absorbiendo un volumen importante de población rural (Rodríguez Martínez, 2008). Ello contribuyó al estancamiento demográfico de las ciudades medias, creando un gran salto entre estas y las capitales de provincia (que no existía históricamente) (Carballo Cortiña, 1977), además del hecho de que las capitales provinciales se llevaron la industria, las dotaciones y el desarrollo (Cuadrado Roura, 1981), provocando que el paro aumentase en las áreas rurales -que veían que con la mecanización del campo la mano de obra sobraba- (Ferrer Rodríguez, 1978) y que llegasen a las cabeceras comarcales ciertas funciones «residuales» de escaso rango (Serrano Martínez, 2005). También determinadas estrategias de transportes e infraestructuras fueron dejando de lado a buena parte de estas ciudades, 
provocando la marginación progresiva de estos núcleos de las vías de comunicación y de comercio (Vilagrasa Ibarz y Ganau Casas, 2003). Ya en los años 80 y 90, el proceso de creación y desarrollo de las autonomías ha dado forma a un sistema de ciudades que gravita casi por completo alrededor de las aglomeraciones capitalinas de primer rango, debilitando de este modo la articulación intraprovincial, sin tampoco mejorar demasiado la interprovincial (Rodríguez Martínez, 2008). Con ello, se ponen de relieve las disparidades existentes entre la fuerte representatividad y centralidad territorial de estos núcleos urbanos y sus escasas posibilidades de desarrollo (Rodríguez Martínez y Sánchez Escolano, 2010a).

Muchas de estas ciudades se engloban en la categoría denominada «agrociudades», cuyo término proviene de las propias características socioeconómicas que estos núcleos poseen (poblaciones que recuerdan las dimensiones de las ciudades pero que poseen muchos elementos que las relacionan con el mundo rural) (López Ontiveros, 1994; Díaz Quidiello, 2007). El peso cuantitativo y cualitativo de este nivel de poblamiento es esencial para la actual y futura organización del territorio (Díaz Quidiello, 2002; Rodríguez Martínez y Sánchez Escolano, 2010a).

\section{III.2. Las ciudades medias andaluzas en la Ordenación del Territorio}

Los gobiernos autonómicos han intentado potenciar, mediante normativas, directrices y planes de ordenación territorial este tipo de ciudades tan característicos de Andalucía (Cano García, 2008), aunque siempre un poco a «remolque» de los cambios y reconsiderando su papel territorial y las responsabilidades que en la gestión del territorio deben tener. El primer intento de desarrollar un territorio articulado aparece en el documento Bases y Estrategias para la ordenación del territorio en Andalucía (Junta de Andalucía, 1999), que precedió al llamado Plan de Ordenación del Territorio de Andalucía (POTA) (Junta de Andalucía, 2006), en el que ya se enmarcara de una manera exacta el conjunto de las ciudades medias dentro de un sistema de ciudades (Rodríguez Martínez y Sánchez Escolano, 2010a). En las Bases y Estrategias los centros intermedios de entre 20.000 y 100.000 habitantes se equiparaban a las ciudades medias y se definían como intermediarios entre el mundo rural y los centros básicos del poblamiento y los centros subregionales, reuniendo en su perfil funcional y en su centralidad el conjunto de funciones y servicios de los núcleos menores de 20.000 habitantes, a los que se suman otros en temas de servicios sanitarios, servicios sociales no básicos, medio ambiente y hacienda pública (Rodríguez Martínez y Sánchez Escolano, 2010a). En algunos años, estos núcleos recuperaron cierto protagonismo gracias a su diversificación productiva y su transformación funcional, en un marco (el del POTA) en el que pasan a ser una de las principales bazas para el desarrollo equilibrado y sostenible de Andalucía. El POTA establece una serie de potencialidades para las ciudades medias: a) el interés patrimonial (ciudades monumentales), b) el ser ejemplo vivo de un sistema urbano típicamente andaluz y mediterráneo y c) la capacidad que tienen para organizar el territorio a partir de su identificación como cabeceras comarcales (Díaz Quidiello, 2007). Aspecto este último que pone de relieve la nueva posición de las ciudades medias como territorios intermedios, entre el centro provincial y los núcleos rurales, y como elementos que cohesionan el territorio y que integran además la propia comarca mediante los servicios y equipamientos que poseen (hospitales, supermercados, algunos centros de ocio...), apoyándose en redes de transporte que favorecen esta conexión (Sánchez Escolano, 2010b). 
En líneas generales, la política territorial andaluza busca un sistema de asentamientos policéntrico, apoyándose en la viabilidad territorial de las ciudades medias como centros comarcales. El POTA reconoce la importancia de las ciudades históricas como las que han configurado el sistema urbano andaluz y señala la necesidad de preservarlas para las siguientes generaciones, como aspecto diferenciador respecto al resto de España y Europa. La necesidad de innovar y ofrecer productos distintos a los derivados de la globalización hace del sistema urbano andaluz un sistema único y difícilmente repetible (Díaz Quidiello, 2007). Sin embargo, existen dos cuestiones criticables en los instrumentos de ordenación territorial: i) se basan únicamente en el criterio demográfico para determinar cuándo un núcleo es una ciudad media (obviando municipios que, pese a no contar con la población necesaria, cuentan con funciones históricas propias de ciudades medias); ii) se determinan los equipamientos y funciones que deben ubicarse en estas ciudades, pero soslayando la financiación, la cual tiene enormes dificultades para llegar hasta esos núcleos (Rodríguez Martínez y Sánchez Escolano, 2010a).

\section{III.3. Selección y delimitación de las áreas metropolitanas andaluzas}

No existe consenso a la hora de definir y delimitar las áreas metropolitanas andaluzas. En el caso andaluz, el Instituto Andaluz de Estadística se refiere al término Aglomeración Urbana como área urbana polinuclear que conforma un mercado unitario de residencia y trabajo, y que refleja a la vez el aumento de escala del espacio de vida colectivo y las diferentes estrategias espaciales de los agentes económicos. Siguiendo el trabajo de Feria Toribio y Susino Arbucias (2005), se ha realizado la delimitación de las áreas metropolitanas andaluzas utilizando los siguientes criterios: movilidad residencia-trabajo, que recoge la interdependencia social/funcional de los espacios (un municipio debe enviar o recibir de otro un flujo superior a 100 personas que además representen más del 15\% de sus ocupados en el primer caso o de sus empleos en el segundo); volumen poblacional mínimo de la ciudad central (100.000 habitantes, de acuerdo a la realidad urbana española y andaluza). Aplicando dichos criterios a los datos del Censo de Población y Vivienda de 2001, se identificaron 8 áreas metropolitanas: siete capitales provinciales (Almería-El Ejido, Bahía de Cádiz, Córdoba, Granada, Huelva, Málaga-Marbella y Sevilla) y Bahía de Algeciras. Jaén, que, aunque como municipio cuenta con el umbral necesario para ser considerado ciudad central, sólo incluye a tres municipios que cumplan los requisitos, siendo un ámbito todavía insuficiente para ser catalogado como área metropolitana.

\section{CLASIFICACIÓN DE LAS CIUDADES MEDIAS ANDALUZAS EN FUNCIÓN DE SU TAMAÑO POBLACIONAL Y SU LOCALIZACIÓN GEOGRÁFICA}

Dado que no todas las ciudades catalogadas como «medias» por su volumen demográfico tienen el mismo comportamiento, es necesario considerar, junto al volumen demográfico, la localización geográfica respecto a los ámbitos más dinámicos: áreas metropolitanas, áreas litorales y áreas interiores.

Según los distintos criterios de diferenciación utilizados, las clasificaciones de ciudades medias andaluzas han variado. En este sentido, algunos autores hablan de ciudad media 
en el contexto andaluz haciendo referencia a asentamientos urbanos, siempre superiores a los 20.000 habitantes -a veces se prefiere el límite de 10.000- y generalmente inferiores a 100.000 habitantes, caracterizados por organizar el territorio en un nivel inferior al de las grandes ciudades, conectando estas últimas con los pequeños asentamientos de carácter rural y desempeñando un papel clave en la articulación regional. De este modo, se establecen diferenciaciones de tipo demográfico (combinándolas a veces con su localización respecto a diferentes redes urbanas y aglomeraciones urbanas) y otros las establecen dependiendo de su ubicación en grandes dominios territoriales (litoral, valle del Guadalquivir, Subbético,...) (ver Fig. 1).

Figura 1

CLASIFICACIÓN DE CIUDADES MEDIAS ANDALUZAS SEGÚN DISTINTOS AUTORES

\begin{tabular}{|l|l|l|}
\hline \multicolumn{1}{|c|}{ Ocaña y Navarro (1999) } & \multicolumn{1}{|c|}{ Cano García (2008) } & \multicolumn{1}{c|}{ Feria Toribio (2012) } \\
\hline -Ciudades medias en entornos & -Ciudades de 20.000-50.000 & -Lugares centrales de nivel \\
metropolitanos. & habs. (divididas a su vez en: & intermedio. \\
-Ciudades medias turísticas o & centros comarcales, ciudades & -Redes de ciudades medias \\
del litoral. & en aglomeraciones urbanas, & (Campiña sevillana, Bajo \\
-Ciudades medias en el espacio & polinucleares y otros tipos). & Guadalquivir, Subbético \\
rural. & -Ciudades de 50.000-100.000 & cordobés). \\
& habs. (subdivididas en las & -Ciudades litorales. \\
& mismas categorías que el tipo & \\
& anterior). & \\
\hline
\end{tabular}

Fuente: Elaboración propia a partir de los autores citados.

Sobre la base de las aportaciones realizadas anteriormente por algunos expertos (Cano García, 2008; Feria Toribio, 2012; Rodríguez Martínez y Sánchez Escolano, 2010a y 2010b), se ha establecido una tipología básica de ciudades medias andaluzas para abordar los objetivos planteados y demostrar nuestras hipótesis. Así, para seleccionarlas se emplea la horquilla de 20.000-100.000 habitantes, según datos del último Censo de Población disponible (2011), incluyendo las ciudades de Dos Hermanas y Marbella a pesar de superar este umbral, pues presentan funcionalidades que las equiparan al resto de ciudades medias, y no son consideradas como centros regionales en el POTA. Junto al umbral de población, se utiliza el criterio de localización geográfica (respecto a las áreas metropolitanas, el litoral y el interior), utilizando el modelo propuesto por Kunzmann (2010), aunque adaptado a la realidad andaluza. Hemos prescindido, pues, de la categoría de ciudades fronterizas, dado que una única ciudad media andaluza (Ayamonte) se localiza en un límite fronterizo internacional difuminado por el espacio Schengen. Por otro lado, las ciudades medias ubicadas en dos categorías (litoral y áreas metropolitanas) han sido clasificadas como ciudades en áreas metropolitanas (ej. Torremolinos), habida cuenta de la mayor influencia del hecho metropolitano respecto al costero. De esta forma, y en función de su localización, las 68 ciudades medias andaluzas que cumplían el criterio demográfico (20.000-100.000 habitantes) fueron clasificadas en tres tipologías: ciudades integradas en áreas metropolitanas (37), ciudades litorales (11) y ciudades de interior (20) (ver tabla 1). 
Tabla 1

CLASIFICACIÓN DE LAS CIUDADES MEDIAS ANDALUZAS SEGÚN SU POBLACIÓN Y LOCALIZACIÓN EN ÁREAS METROPOLITANAS, DE LITORAL Y DE INTERIOR

\begin{tabular}{|c|c|c|}
\hline MUNICIPIO & POBLACIÓN 2011 & LOCALIZACIÓN RELATIVA \\
\hline Adra & 24.375 & Áreas Metropolitanas \\
\hline Alcalá de Guadaira & 73.317 & Áreas Metropolitanas \\
\hline Alhaurín de la Torre & 37.020 & Áreas Metropolitanas \\
\hline Alhaurín el Grande & 23.164 & Áreas Metropolitanas \\
\hline Armilla & 22.593 & Áreas Metropolitanas \\
\hline Barrios (Los) & 22.988 & Áreas Metropolitanas \\
\hline Benalmádena & 61.394 & Áreas Metropolitanas \\
\hline Bormujos & 20.200 & Áreas Metropolitanas \\
\hline Camas & 26.433 & Áreas Metropolitanas \\
\hline Carmona & 28.817 & Áreas Metropolitanas \\
\hline Cártama & 23.225 & Áreas Metropolitanas \\
\hline Chiclana de la Fra. & 80.769 & Áreas Metropolitanas \\
\hline Coín & 21.692 & Áreas Metropolitanas \\
\hline Coria del Río & 29.880 & Áreas Metropolitanas \\
\hline Dos Hermanas & 128.433 & Áreas Metropolitanas \\
\hline Ejido (El) & 80.839 & Áreas Metropolitanas \\
\hline Estepona & 64.468 & Áreas Metropolitanas \\
\hline Fuengirola & 72.019 & Áreas Metropolitanas \\
\hline Línea, la & 65.412 & Áreas Metropolitanas \\
\hline Mairena del Alcor & 21.895 & Áreas Metropolitanas \\
\hline Mairena del Aljarafe & 42.570 & Áreas Metropolitanas \\
\hline Maracena & 21.560 & Áreas Metropolitanas \\
\hline Marbella & 135.124 & Áreas Metropolitanas \\
\hline Mijas & 74.028 & Áreas Metropolitanas \\
\hline Moguer & 20.668 & Áreas Metropolitanas \\
\hline Palacios y Villafranca (Los) & 37.720 & Áreas Metropolitanas \\
\hline Puerto de Santa María (El) & 89.012 & Áreas Metropolitanas \\
\hline Puerto Real & 41.299 & Áreas Metropolitanas \\
\hline Roquetas de Mar & 86.799 & Áreas Metropolitanas \\
\hline Rota & 29.169 & Áreas Metropolitanas \\
\hline San Fernando & 96.786 & Áreas Metropolitanas \\
\hline San Juan de Aznalfarache & 21.484 & Áreas Metropolitanas \\
\hline San Roque & 29.956 & Áreas Metropolitanas \\
\hline Tomares & 23.938 & Áreas Metropolitanas \\
\hline Torremolinos & 66.270 & Áreas Metropolitanas \\
\hline Utrera & 51.722 & Áreas Metropolitanas \\
\hline Vícar & 23.482 & Áreas Metropolitanas \\
\hline Almonte & 22.243 & Litoral \\
\hline
\end{tabular}




\begin{tabular}{|c|c|c|}
\hline Almuñécar & 26.969 & Litoral \\
\hline Ayamonte & 20.540 & Litoral \\
\hline Conil de la Frontera & 21.755 & Litoral \\
\hline Isla Cristina & 21.844 & Litoral \\
\hline Lepe & 26.538 & Litoral \\
\hline Motril & 60.460 & Litoral \\
\hline Nerja & 21.086 & Litoral \\
\hline Níjar & 28.223 & Litoral \\
\hline Sanlúcar de Barrameda & 67.232 & Litoral \\
\hline Vélez-Málaga & 76.922 & Litoral \\
\hline Alcalá la Real & 22.722 & Interior \\
\hline Andújar & 38.813 & Interior \\
\hline Antequera & 41.741 & Interior \\
\hline Arcos de la Frontera & 31.368 & Interior \\
\hline Baena & 20.061 & Interior \\
\hline Baza & 21.276 & Interior \\
\hline Cabra & 21.085 & Interior \\
\hline Écija & 40.630 & Interior \\
\hline Lebrija & 27.241 & Interior \\
\hline Linares & 60.799 & Interior \\
\hline Loja & 21.431 & Interior \\
\hline Lucena & 42.355 & Interior \\
\hline Martos & 24.457 & Interior \\
\hline Montilla & 23.797 & Interior \\
\hline Morón de la Fra. & 28.389 & Interior \\
\hline Palma del Río & 21.454 & Interior \\
\hline Priego de Córdoba & 23.408 & Interior \\
\hline Puente Genil & 30.304 & Interior \\
\hline Ronda & 36.473 & Interior \\
\hline Úbeda & 35.622 & Interior \\
\hline
\end{tabular}

Fuente: elaboración propia a partir de datos del Censo de Población (2011).

En el mapa 1 aparecen representados los límites de las áreas metropolitanas existentes en Andalucía y la localización de las 68 ciudades medias, distinguiendo entre las 3 tipologías señaladas anteriormente (dentro de áreas metropolitanas, en el litoral y en el interior).

Esta clasificación permite establecer subgrupos dentro de cada una de las tres tipologías. A continuación, se analiza el significado de esos tipos y subtipos:

A) Ciudades medias integradas dentro de áreas metropolitanas. Su comportamiento es peculiar debido a que están impregnadas del hecho metropolitano y sus dinámicas. Son núcleos condicionados por las dinámicas generadas desde o hacia los grandes centros regionales. En las últimas décadas, han experimentado un crecimiento demográfico superior a la 
media al ser receptoras de población procedente de la ciudad central y han sido objeto de procesos de periurbanización, rururbanización y urbanización difusa, situaciones acompañadas generalmente de un fuerte boom inmobiliario.

A.1. Ciudades con urbanismo disperso. Son eminentemente rurales y tenían escaso peso demográfico en los años 60 y 70, aunque han crecido de manera desproporcionada durante las últimas tres décadas debido al uso masivo del vehículo privado, a cambios en las preferencias por viviendas unifamiliares (de menor precio que en las grandes ciudades) y al desarrollo industrial y de otras actividades (comerciales, logísticas). Actualmente presentan características funcionales de periferias metropolitanas (en áreas metropolitanas como Sevilla o Málaga) o de núcleos periurbanos (en el resto). Es el caso de municipios del Aljarafe (Mairena del Aljarafe, San Juan de Aznalfarache, Bormujos o Tomares) y de otros sectores del área metropolitana de Sevilla (Alcalá de Guadaira, Dos Hermanas), de Málaga (Alhaurín de la Torre, Benalmádena, Torremolinos,...) y Granada (Maracena). En todos los casos el proceso se acompaña por un fuerte aumento de la población residente -asociado a pirámides de población con elevada presencia de estratos jóvenes-; en algunas ciudades se suma a ello un incremento del empleo y una buena posición en indicadores que identifican procesos de innovación empresarial.

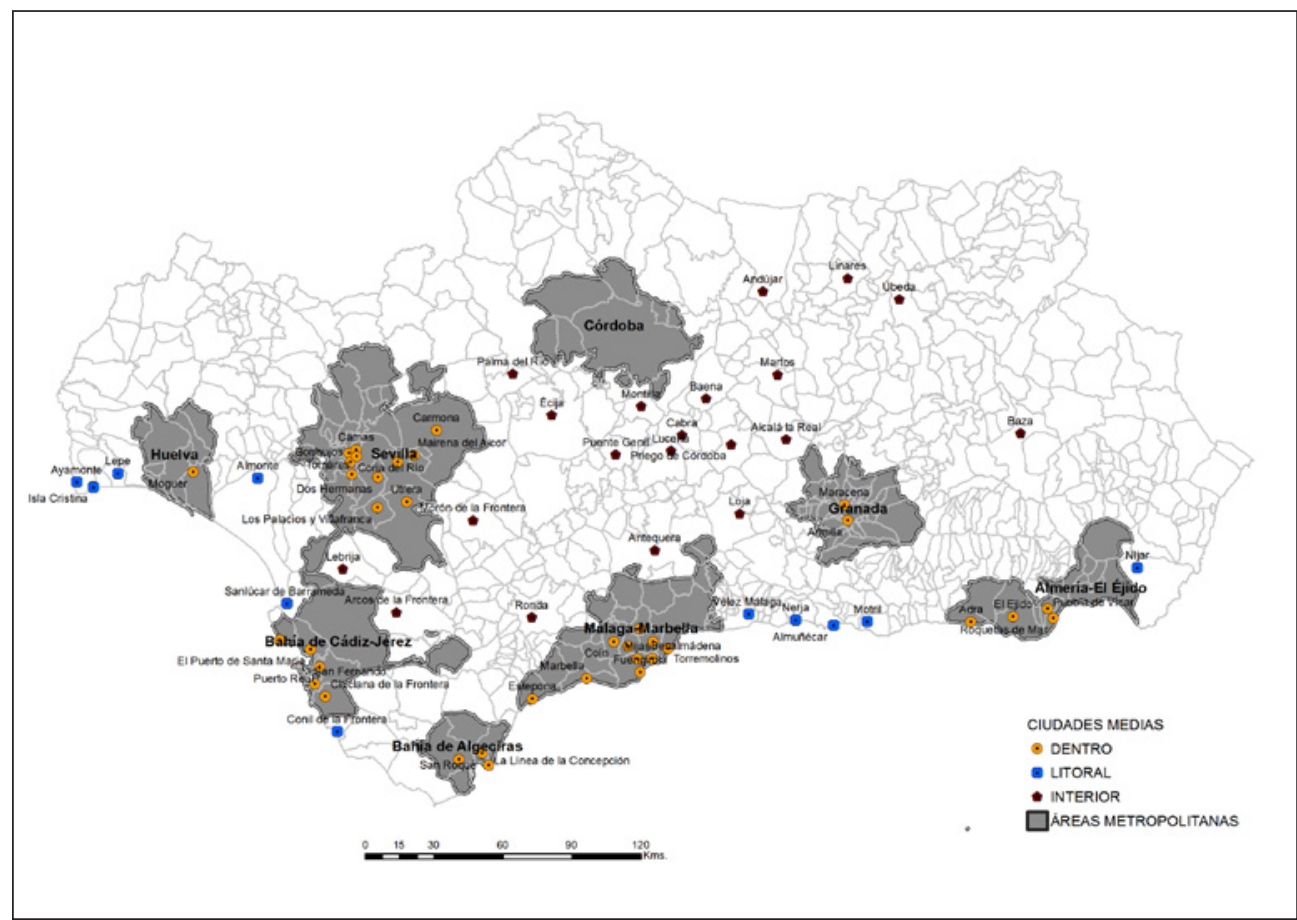

Fuente: elaboración propia. 
A.2. Ciudades de carácter industrial. Este subtipo incluye las ciudades que sirvieron de asiento a la implantación de grandes empresas exógenas, con un acusado predominio de sectores de cabecera y fuerte impacto ambiental, factorías de elevada dimensión y cuantiosos efectivos laborales (caso de los núcleos de la Bahía de Algeciras: La Línea, Los Barrios y San Roque). En el otro gran foco industrial del área metropolitana Bahía de Cádiz (Puerto Real y San Fernando), la crisis del sector naval y sus consecuencias resultan visibles y alejan a sus ciudades de las mejores posiciones en la mayoría de indicadores.

B) Ciudades medias situadas en el litoral. Se han beneficiado de una particular dinámica económica determinada por el auge del sector turístico y la construcción, y, en muchos casos, cuentan con un importante desarrollo de una agricultura intensiva de cultivos bajo plástico. El boom de estos sectores económicos -casi siempre especulativos- ha hecho que estas ciudades se desarrollen rápidamente, atrayendo a un gran número de inmigrantes y aumentando las rentas disponibles. Esa inmigración va unida al crecimiento vegetativo y a la llegada de extranjeros que buscan lugares de residencia de clima más favorable. Alejadas de la influencia de las áreas metropolitanas, pero beneficiadas de su localización litoral, algunas han desarrollado un turismo de menor densidad y mayor calidad (Ayamonte, Sanlúcar de Barrameda, Motril, Nerja o Níjar). Otras, enclavadas dentro de áreas metropolitanas, se han beneficiado tanto de su cercanía a la ciudad principal y a infraestructuras de transporte de primer nivel (aeropuertos, alta velocidad ferroviaria o carreteras de gran capacidad) como de su localización costera, constituyendo importantes y más densas áreas turísticas. Se trata de ciudades como Fuengirola, Benalmádena y Torremolinos (área metropolitana de Málaga); Adra, El Ejido, Roquetas de Mar y Níjar (área metropolitana de Almería); Lepe, Ayamonte, Isla Cristina y Moguer (área metropolitana de Huelva). En muchas de estas ciudades se ha producido un fenómeno peculiar de conurbación que se ha ido conformando en las últimas décadas (especialmente en el litoral mediterráneo). La dinámica de estos núcleos ha elevado el nivel de los indicadores relativos a dotaciones de equipamientos e infraestructuras -a pesar de la crisis y la reducción de las inversiones públicas, que sí han relegado a un papel secundario las dotaciones de carácter más social-, los niveles de motorización o la cualificación media de sus recursos humanos.

C) Ciudades medias de interior. Son ciudades herederas de las antiguas «agrociudades» ubicadas en su mayoría en las campiñas, el valle del Guadalquivir y el Surco Intrabético y que mantienen ciertas características económicas y socioculturales propias, en buena medida, del mundo rural (López Ontiveros, 1994). La mayoría de ellas se encuentran en una situación periférica respecto a las áreas metropolitanas y litorales -posición más desfavorable en cuanto al crecimiento demográfico y económico y que puede provocar ausencia de interés en lo que respecta a las inversiones, tanto públicas como privadas-, aunque a menudo los indicadores ambientales y sociales presentan niveles más elevados y saludables. Han experimentado menos transformaciones, conservando una importante herencia histórica y manteniendo un papel intermediario entre el nivel urbano-metropolitano y el ámbito rural (Díaz Quidiello, 2002). Estas ciudades han experimentado durante los últimos años una estabilización de su crecimiento (cuando no un cierto freno o regresión) y, aunque se ha producido un trasvase de la población del sector primario al secundario y, sobre todo, al terciario, las transformaciones socioeconómicas no han sido ni tan rápidas ni tan radicales. Ello ha provocado que aún se 
conserve su esencia como antiguas «agrociudades» y también su patrimonio. En estos casos, las dinámicas especulativas no han sido tan fuertes como para destruir o deteriorar su patrimonio, sus tradiciones y su acervo cultural, que se convierten en potencialidades y fortalezas de estas ciudades. Su debilidad es, quizá, un cierto abandono en los últimos tiempos por parte de las Administraciones Públicas, que no las consideran prioritarias a la hora de realizar inversiones en equipamientos e infraestructuras. Tras una época álgida en que afluían fondos europeos (FEDER o FEOGA) que hicieron de ellas lugares atractivos, con buenos servicios y atrayentes para la población, hoy han entrado en cierta decadencia. A pesar del reconocimiento de su papel articulador y organizador dentro de los instrumentos de gestión territorial, en la práctica han quedado al margen de algunas políticas e inversiones en equipamientos e infraestructuras. A pesar de haber desempeñado un papel relevante en la historia del territorio andaluz, se han quedado aisladas de los ámbitos más dinámicos (metropolitanos y litorales) y relegadas a una posición secundaria. De no modificarse este planteamiento, estas ciudades pueden entrar en una deriva de regresión que les impediría cumplir su rol y que provocaría una polarización entre grandes conjuntos urbanos y pequeños asentamientos rurales. En ese ámbito territorial existen, no obstante, algunas excepciones identificadas, muchas de ellas, con pequeñas ciudades que cuentan con sistemas productivos locales especializados e innovadores, lo que les permite posicionarse mejor desde el punto de vista socioeconómico. Son, por ejemplo, los casos de Lucena, Cabra, Baena, Andújar, Linares, Úbeda, Écija, Morón de la Frontera, Antequera y Ronda.

En los gráficos 1 y 2 se presenta la evolución de la población de las ciudades medias desde 1860 hasta 2011, distinguiéndolas según su localización en áreas metropolitanas, en el litoral o en el interior. Concretamente en el gráfico 1 se observa la evolución poblacional en datos absolutos, destacando cómo, si bien hasta 1950 las ciudades medias de interior concentraban un mayor volumen de población que el resto de ciudades medias, es a partir

Gráfico 1

EVOLUCIÓN DE LA POBLACIÓN DE LAS CIUDADES MEDIAS ANDALUZAS SEGÚN SU LOCALIZACIÓN (1860-2011)

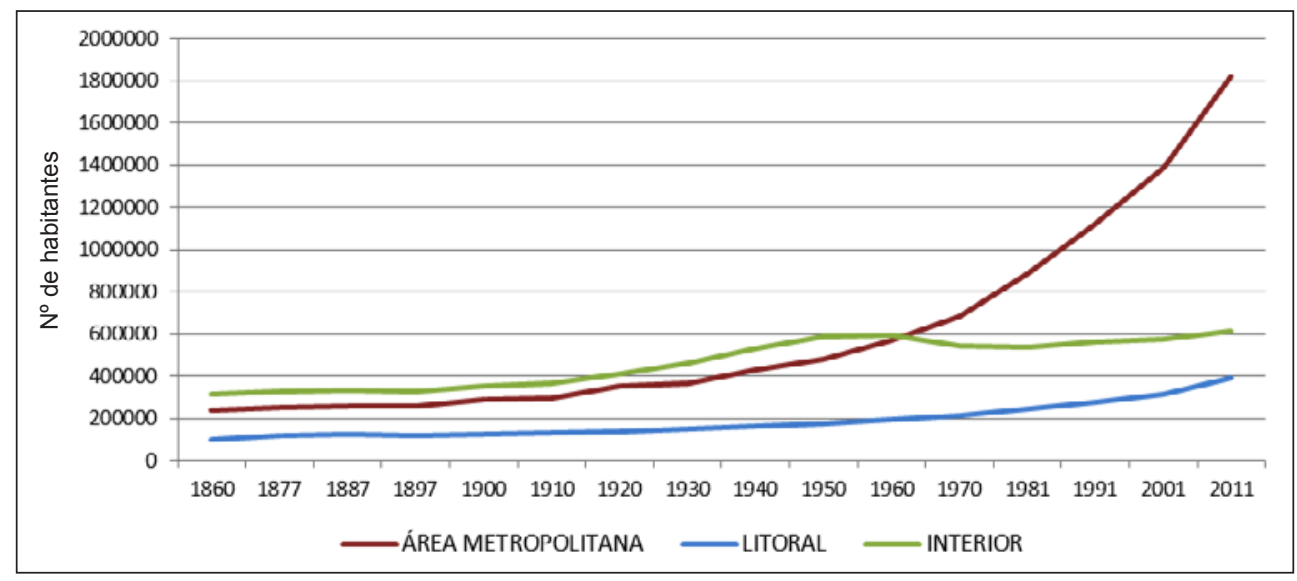

Fuente: elaboración propia con datos del Censo de Población (1860-2011). 
de esta fecha cuando comienza su estancamiento demográfico, entrando incluso en una recesión (años 60 y 70), frente al aumento de la población de las ciudades medias situadas en áreas metropolitanas y, en menor medida, de las ciudades medias del litoral a partir de los años 70 .

Por su parte, en el gráfico 2 aparecen representados los porcentajes de crecimiento demográfico en las tres tipologías de ciudades medias. De estos datos podemos deducir: a) las ciudades medias en áreas metropolitanas crecen de manera discontinua hasta los años 40, aunque a partir de dicha década lo harán de manera constante, con niveles de crecimiento cercanos al $20 \%$ a partir de los años 60 y 70, cercanos al 30\% en los años 80 y 90, o incluso superiores al $30 \%$ en 2011 ; b) las ciudades medias del litoral experimentan crecimientos bajos hasta los años 30, aunque registran grandes crecimientos a partir de los años 60 y especialmente en la última década (con crecimientos del $13 \%$ y del $25 \%$ ); c) las ciudades medias ubicadas en el interior, cuyos mayores crecimientos se registran entre los años 20 y 50 , comienzan a aletargarse a partir de los 60, produciéndose incluso retrocesos o caídas en los 70 y 80, con una ligera recuperación en las últimas décadas.

Gráfico 2

EVOLUCIÓN PORCENTUAL DE LA POBLACIÓN DE LAS CIUDADES MEDIAS ANDALUZAS SEGÚN SU LOCALIZACIÓN (1860-2011)

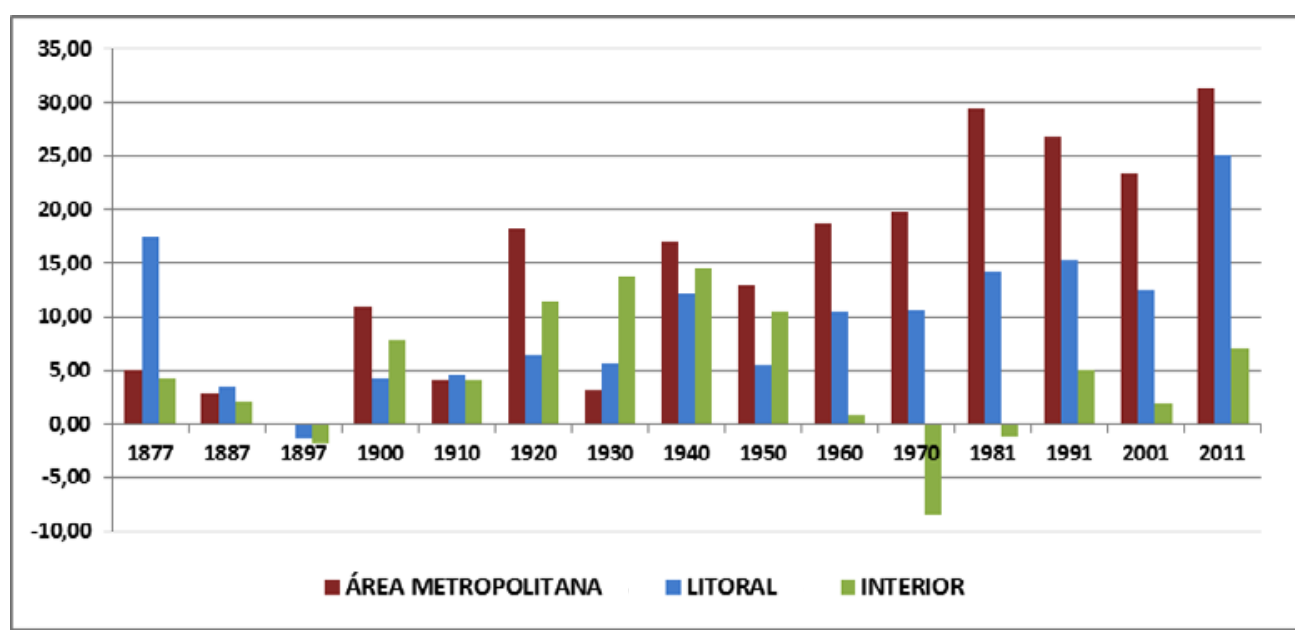

Fuente: elaboración propia con datos del Censo de Población (1860-2011).

Como se pone de manifiesto, los cambios de índole demográfica son dispares dependiendo de su localización. Así, las ciudades medias localizadas en áreas metropolitanas se ven beneficiadas del fenómeno metropolitano, siendo su comportamiento más fruto de las transformaciones del conjunto que de un comportamiento individual o local. Por su parte, las pertenecientes al litoral se han visto también envueltas en grandes cambios socioeconómicos y territoriales (boom inmobiliario por el sobredimensionamiento del sector turístico). Frente a tales situaciones, las ciudades medias de interior, aun reconociéndoseles su papel articula- 
dor territorial y como lugares centrales, han quedado marginadas de muchas de las políticas territoriales, de infraestructuras y de servicios públicos acometidas en las últimas décadas.

La existencia de esos diferentes modos de desarrollo de las ciudades medias, en función de su localización respecto de las áreas metropolitanas y litorales, puede explicarse por el desarrollo de dos tipos de dinámicas: una, de tipo espontáneo: dinámica económica, en función de la tendencia del mercado económico y de las preferencias de los ciudadanos; otra, inducida por los poderes públicos: inversiones, infraestructuras de transporte, equipamientos sanitarios, etc. En este sentido, y enlazando con esta segunda dinámica, podemos destacar algunos hitos de importancia: i) se desmantelan líneas de ferrocarril convencionales que unen ciudades medias interiores mientras se realizan grandes inversiones en infraestructuras de transporte para dotar a las grandes urbes y/o ciudades medias situadas en áreas metropolitanas o litorales (Ej. alta velocidad ferroviaria, metros en las ciudades de Sevilla, Málaga o Granada, tranvías de Bahía de Cádiz, Sevilla, Jaén, Aljarafe, Dos Hermanas o Alcalá de Guadaira); ii) se produce un freno a la inversión en servicios y equipamientos como hospitales comarcales y centros educativos, pues, aunque hubo un momento de auge principalmente desde inicios de los años 80, la inversión en este tipo de elementos de bienestar social ha experimentado una contracción drástica, provocando que la oferta de tales instalaciones empiece a ser insuficiente en determinadas comarcas (Ej. se reducen las prestaciones de los hospitales comarcales con el objetivo teórico de racionalizar los recursos sanitarios públicos); iii) se desarrollan los primeros planes de ámbito subregional centrándose en áreas metropolitanas y litorales, de modo que la ordenación del territorio parece dejar de lado a las ciudades medias interiores.

\section{CONCLUSIONES}

Las ciudades medias andaluzas de interior poseen una serie de características que las hacen atractivas desde diversos puntos de vista. En primer lugar, acumulan un importante patrimonio basado en la identidad, personalidad y singularidad de sus tradiciones, lo cual habla de su importancia histórica como centros de decisión y plantea la necesidad de reconocer, proteger y potenciar tal riqueza como pieza importante para el desarrollo de un turismo urbano sostenible, incorporando a estas ciudades dentro de las rutas turísticas regionales (Muñoz Martínez, 2007; Merinero Rodríguez y Lara de Vicente, 2011). En segundo lugar, suponen espacios de descongestión de las áreas metropolitanas en contraposición a los saturados centros urbanos, proporcionando oportunidades residenciales más económicas en espacios que disfrutan de una menor congestión de tráfico, ambientes más saludables y mejor cohesión social.

No obstante, la falta de inversiones públicas en nuevas infraestructuras de transporte en estas ciudades medias, unida a su concentración dentro de las áreas metropolitanas (aeropuertos, estaciones de alta velocidad ferroviaria, tranvía metropolitano, metro, etc.), aleja aún más a este tipo de ciudades de los centros de decisión. Se hace por ello necesario potenciar la red convencional de ferrocarriles y los trenes de media distancia, aumentando la conectividad de estas ciudades. Esta carencia de inversión a la que aludimos provoca también una disminución de servicios y equipamientos de salud, educación y sociales, lo que repercute sin duda en el bienestar y calidad de vida de sus habitantes. Esta situación se hace más problemática 
por las escasas oportunidades laborales en estas ciudades, que provocan que la población más dinámica, compuesta generalmente por jóvenes con mayor cualificación, se vea obligada a emigrar a áreas metropolitanas o a otras regiones. Otro elemento desfavorable es su fuerte dependencia del sector público, las subvenciones y las ayudas externas, lo que, junto a la ausencia de tejido empresarial, fomenta un desarrollo económico escasamente competitivo. Para atraer inversiones que permitan un nuevo desarrollo de estas ciudades se requeriría la revitalización de su imagen mediante la puesta en valor de su localización y emplazamiento, destacando su calidad de vida y ambiental, utilizando instrumentos de marketing urbano y planificación estratégica. Junto a estas actuaciones, se requieren actitudes innovadoras que posibiliten la puesta en valor de los recursos y la asunción colectiva de un proyecto de ciudad (Caravaca Barroso y González Romero, 2010; Caravaca Barroso et al., 2014).

Nos encontramos en definitiva ante la potenciación de las grandes urbes y áreas metropolitanas, mientras se aboca a las ciudades medias interiores, localizadas al margen de los ámbitos metropolitanos y litorales, a una lenta decadencia. A este proceso se deberá hacer frente a través de medidas nacionales y regionales (económicas, infraestructurales, territoriales), si no queremos que nuestro mundo rural y de interior quede olvidado y apartado de los grandes ejes de crecimiento. Esta situación podría ser resuelta mediante la aplicación de los instrumentos de planificación territorial existentes, que otorgan funciones, servicios y equipamientos específicos a cada nivel jerárquico urbano para su implementación y desarrollo. Pese a ello, diversos autores han señalado que estas actuaciones han formado parte más del discurso político que de las actuaciones realmente implementadas, poniéndose de manifiesto una gran discordancia entre las declaraciones públicas, aparentemente orientadas al desarrollo de las ciudades medias de interior y de las áreas rurales, y una realidad, ya sea espontánea (fuerzas del mercado), ya sea inducida por los poderes públicos (inversiones), que sigue propiciando el desarrollo desmesurado de las grandes urbes, los ámbitos metropolitanos y las áreas litorales, lo cual conlleva graves consecuencias para el equilibrio territorial regional.

\section{BIBLIOGRAFÍA}

AMORIM FILHO, O. y SERRA, R.V. (2001): Evolução e perspectivas do papel das cidadesmédias no planejamento urbano e regional. Cidades médias brasileiras. Rio de Janeiro, IPEA, pp. 1-34.

ANDRÉS LÓPEZ, G. (2008): «Geografía y ciudades medias en España: ¿a la búsqueda de una definición innecesaria?». Scripta Nova, vol. XII, núm. 270 (49). http://www.ub.es/ geocrit/sn/sn-270/sn-270-49.htm

BELLET SANFELIU, C. y LLOP TORNÉ, J.M. (1999): «Ciudades intermedias y urbanización mundial. Presentación del programa de trabajo de la Unión Internacional de Arquitectos (UIA)» en Ciudades intermedias y urbanización mundial (Bellet Sanfeliu, C. y Llop Torné J.M., coord.). Lleida, Editorial Milenio, pp. 325-347.

CANO GARCÍA, G. (2008): «Clasificaciones urbanas en Andalucía. Las ciudades medias». Revista de Estudios Andaluces, ${ }^{\circ}$ 27, pp. 115-153.

CARAVACA BARROSO, I. y GONZÁLEZ ROMERO, G. (2010): «Estrategias y actuaciones para el desarrollo de ciudades medias. Algunos ejemplos». Scripta Nova, vol. XIV, $\mathrm{n}^{\circ}$ 331 (33). http://www.ub.es/geocrit/sn/sn-331/sn-331-33.htm 
CARAVACA BARROSO, I. et al. (2014): «Conocimiento, innovación y estrategias públicas de desarrollo: análisis comparado de tres ciudades medias de Andalucía (España)». EURE, vol. 40, n 119, pp. 49-74.

CARBALLO CORTIÑA, R. (1977): Capitalismo y agricultura en España: La evolución de las relaciones de producción en el campo (1939/75). Madrid, Ediciones de la Torre.

CUADRADO ROURA, J.R. (1981): La Política Regional en los planes de desarrollo (19641975). La España de las Autonomías, pasado, presente y futuro. Madrid, Espasa Calpe.

DÍAZ QUIDIELLO, J.L. (2002): «Las ciudades medias históricas», Boletín del Instituto Andaluz del Patrimonio Histórico, no 38, pp. 204-209.

DÍAZ QUIDIELLO, J.L. (2007): «Las ciudades medias interiores en el Plan de Ordenación del Territorio de Andalucía». Boletín del Instituto Andaluz del Patrimonio Histórico, $\mathrm{n}^{\circ}$ 63, pp. 44-52.

ENTRENA DURÁN, F. (2004): «El fenómeno de la periurbanización en Europa», Jornades Europees d'Agricultura Periurbana. Barcelona, pp. 13.

ESTEBAN, A. y LÓPEZ, A. (1989): «El papel de las ciudades medias en España. Presente y futuro». Urbanismo, no 6, pp. 6-16.

EUROPEAN FOUNDATION FOR THE IMPROVEMENT OF LIVING AND WORKING CONDITIONS, ed. (1994): Visions and Actions for Medium -Size Cities. Luxembourg.

EUROPEAN SPATIAL PLANNING OBSERVATION NETWORK - ESPON (2006): The Role of Small and Medium-Sized Towns (SMESTO). Viena, European Commission. Disponible en:

http://www.espon.eu/export/sites/default/Documents/Projects/ESPON2006Projects/StudiesScientificSupportProjects/SmallMediumCities/fr-1.4.1_revised-full.pdf

EUROPEAN SPATIAL PLANNING OBSERVATION NETWORK - ESPON (2012): TOWN Small and medium sized towns in their functional territorial context. Luxemburgo: European Commission. Disponible en: http://www.espon.eu/export/sites/default/Documents/ Projects/AppliedResearch/TOWN/TOWN_Inception_report_July_2012.pdf

FERIA TORIBIO J.M. y SUSINO ARBUCIAS J. (2005): Movilidad por razón de trabajo en Andalucía 2001. Sevilla, Instituto de Estadística de Andalucía.

FERIA TORIBIO J.M. (2012): «La ordenación del territorio en las áreas metropolitanas españolas» en Ordenación del Territorio y Urbanismo: conflictos y oportunidades (Jurado Almonte J. M., coord.). Sevilla, Universidad Internacional de Andalucía: pp. 127-158.

FERRER RODRÍGUEZ,A. (1978): «La mecanización del campo de Andalucía». Cuadernos Geográficos de la Universidad de Granada, nº 8, pp. 117-130.

GARCÍA MONTALVO, J. (2006): «Deconstruyendo la burbuja: expectativas de revalorización y precio de la vivienda en España». Papeles de economía española, $\mathrm{n}^{\circ} 109$, pp. 44-75.

GARCÍA SANZ, B. y MARTÍNEZ PARICIO, J.I. (1998): Envejecimiento en el mundo rural: problemas y soluciones. Madrid, Ministerio de Trabajo y Asuntos Sociales.

GRAÑA, F.M. (2002): Creación de empresas. Factores asociados al éxito emprendedor en cinco ciudades argentinas. Buenos Aires, Editorial Martín.

JUNTA DE ANDALUCÍA (1999): Bases y Estrategias del Plan de Ordenación del Territorio de Andalucía. Sevilla, Junta de Andalucía. 
JUNTA DE ANDALUCÍA (2006): Plan de Ordenación del Territorio de Andalucía. Sevilla, Junta de Andalucía.

KUNZMANN, K.R. (2010): «Medium-Sized Towns, Strategic Planning and Creative Governance» en Making Strategies in Spatial Planning, 27 Urban and Landscape Perspectives Vol. 9 (Cerreta et al., Eds.). Berlin, Springer.

LAJUGIE, J. (1974): Les villes moyennes. París, Cujas.

LLOP TORNÉ, J.M. (1999): Ciudades intermedias y urbanización mundial. Lleida, UNESCO.

LÓPEZ LARA, E. (2015): «El valor estratégico de los servicios en la construcción del territorio» en El papel de los servicios en la construcción del territorio: redes y actores (Espinosa Seguí, A.I. y Antón Burgos, F.J. (Eds.). Madrid, Asociación de Geógrafos Españoles. Tomo I, pp. 201-222.

LÓPEZ ONTIVEROS, A. (1994): «La agrociudad andaluza: Caracterización, estructura y problemática». Estudios Regionales, no 39, pp. 59-91.

MACÍAS MACÍAS, A. (2004): «Crecimiento económico y competitividad en las regiones, las ciudades medias de Jalisco: el caso de Zapotlán El Grande». Región y sociedad, vol. 16 (31), pp. 39-82.

MARQUES DA COSTA, E. (2002): «Ciudades médias: contributos para a sua definição». Finisterra: Revista Portuguesa de Geografia, 37 (74), pp. 101-128.

MERINERO RODRÍGUEZ, R. y LARA DE VICENTE, F. (2011): «Las Ciudades Medias del interior de Andalucía. Caracterización y retos para el desarrollo turístico en un nuevo entorno». Políticas Activas en Turismo. Respuestas a la singularidad del mercado laboral, 9 - 11 Noviembre 2011. XVI Congreso AECIT.

MUÑOZ MARTÍNEZ, A. (2007): «El Plan Turístico de Ciudades Medias: el interior avanza». Boletín del Instituto Andaluz del Patrimonio Histórico, $\mathrm{n}^{\circ}$ 63, pp. 70-74.

OCAÑA OCAÑA, C. y NAVARRO RODRÍGUEZ, S. R. (1999): «Núcleos de población de volumen medio en Andalucía» en La ciudad: tamaño y crecimiento (Domínguez Rodríguez, R.). Málaga, Universidad de Málaga pp. 133-143.

PASCIARONI, C. (2012): «Ciudades medias: Aproximación Metodológica, Funcionalidades y Estructura Productiva». Ciencias Económicas, vol. 30 (1), pp. 399-415.

RIVKIN, GOLDIE W. AND LACOLM D. RIVKIN (1982): Approaches to Planning for Secondary Cities in Developing Countries. Study for US Aid Washington.

RODRÍGUEZ MARTÍNEZ, F. y SÁNCHEZ ESCOLANO, L.M. (2010a): «Cambios en el Sistema Urbano intermedio de Andalucía: Significado geográfico y nueva funcionalidad urbana». X Coloquio y Jornadas de Campo de Geografía Urbana, 28 Junio - 3 Julio 2010, Congreso de Geografía Urbana. AGE, pp. 496-507.

RODRÍGUEZ MARTÍNEZ, F. y SÁNCHEZ ESCOLANO, L.M. (2010b): «Sobre la nueva dimensión territorial de las ciudades medias en Andalucía» en Ciudad, territorio y paisaje: Reflexiones para un debate multidisciplinar (Cornejo Nieto, C. et al., coords.), Madrid, Universidad Autónoma, pp. 272-287.

RODRÍGUEZ MARTÍNEZ, F. (2008): «Las ciudades medias andaluzas. Cambios básicos durante el último medio siglo», Homenaje a Joaquín Bosque Maurel: Secretario General de la Real Sociedad Geográfica (1983-2008), Real Sociedad Geográfica, pp. 199-238. 
ROMERO GONZÁLEZ, J. (2005): «El gobierno del territorio en España. Balance de iniciativas de coordinación y cooperación territorial». Boletín de la Asociación de Geógrafos Españoles, n 39, pp. 59-86.

RONDINELLI, D. (1983): Secondary cities in developing countries. Beverly Hills, Sage Publications Inc.

SÁEZ PÉREZ, L.A., PINILLA NAVARRO, V.J., AYUDA BOSQUE, M.I. (2001): «Políticas ante la despoblación en el medio rural: un enfoque desde la demanda». Ager: Revista de estudios sobre despoblación y desarrollo rural, $\mathrm{n}^{\circ} 1$, pp. 211-232.

SÁNCHEZ ESCOLANO, L.M. (2010): «Las redes de ciudades medias en la provincia de Granada: transformaciones recientes y nuevas tipologías (1950-2008)». Cuadernos Geográficos de la Universidad de Granada, $\mathrm{n}^{\circ}$ 46, pp. 111-138.

SATTERTHWAITE, D. y TACOLI, C. (2003): «The urban part of rural development: the role of small and intermediate urban centres in rural and regional development and poverty reduction». International Institute for Environment and Development (IIED).

SERRANO MARTÍNEZ, J.M. (2005): «Ciudades pequeñas y medias centros de comarca. Análisis de la región de Murcia» en La ciudad: tamaño y crecimiento (Domínguez Rodríguez, R.). Málaga, Universidad de Málaga, pp. 171-186.

SPOSITO, M.E. (2004): «Novos conteúdos nas periferias urbanas das cidades médias do Estado de São Paulo, Brasil». Investigaciones Geográficas, nº 54, pp. 114-139.

VILAGRASA IBARZ, J. y GANAU CASAS, J. (2003): «Ciudades medias en España: posición en la red urbana y procesos urbanos recientes». Mediterráneo económico, $\mathrm{n}^{\circ} 3$, pp. 37-73.

VILAGRASA IBARZ, J. (1999): «Las ciudades pequeñas y medias en España» en $L a$ ciudad: tamaño y crecimiento 1999 (Domínguez, R. Coord.), Universidad de Málaga (UMA) y Asociación de Geógrafos Españoles, pp. 17-49.

VILAGRASA IBARZ, J. (2000): Ciudades medias y ciudades intermedias: posicionamiento en la red urbana y procesos urbanos recientes. Lleida, Universitat de Lleida. 
